\title{
The Contact Resistance Performance of Gold Coated Carbon-Nanotube Surfaces under Low Current Switching
}

\author{
John W. McBRIDE ${ }^{1,2}$, Chamaporn CHIANRABUTRA ${ }^{1}$, Liudi JIANG ${ }^{1}$, and Suan Hui PU ${ }^{2}$ \\ Faculty of Engineering and the Environment \\ ${ }^{1}$ University of Southampton, Southampton SO17 1BJ, UK \\ ${ }^{2}$ University of Southampton Malaysia Campus, Johor, 79200, Malaysia \\ E-mail: jwm@soton.ac.uk
}

\begin{abstract}
Multi-Walled CNT (MWCNT) are synthesized on a silicon wafer and sputter coated with a gold film. The planar surfaces are mounted on the tip of a piezo-electric actuator and mated with a gold coated hemispherical surface to form an electrical contact. These switching contacts are tested under conditions typical of MEMS relay applications; $4 \mathrm{~V}$, with a static contact force of $1 \mathrm{mN}$, at a low current between $20-50 \mathrm{~mA}$. The evolution of contact resistance is considered in a newly developed test procedure. The contact resistance performance is then linked to a study of the contact changes in the surface. The contact surfaces have been shown to exhibit a transfer process over a large number of switching cycles. The continuous monitoring of contact resistance can be used for the identification of surface failure. The results show that for the surfaces presented the contact resistance remains stable for between 80 and 120 million switching cycles. Interestingly the number of bounces is related to the fine transfer failure mechanism.
\end{abstract}

Keyword Carbon nanotubes, contact surface, MEMS switching surface, fine transfer mechanism, contact resistance.

\section{Introduction}

In MEMS relay devices, a stable contact resistance $\left(\mathrm{R}_{\mathrm{c}}\right)$ of less than 1-2 $\Omega$ is required over millions of switching cycles [1]. To meet this specification a number of materials have been used for the switch contact surfaces, for example palladium alloys, platinum alloys, and gold [1]. Although these materials have low resistance, they are soft and wear easily. Silicon carbide and diamond have also been investigated because of the high elastic moduli, but these materials are limited in their use for electrical contact applications due to low conductivity.

Carbon nanotubes (CNTs) have both good electrical and mechanical properties [2], making them attractive for contact surfaces for a wide range of applications, including MEMS relays. CNTs have two main structures: single-walled nanotubes (SWCNTs) and multi-walled nanotubes (MWCNTs). MWCNTs are relatively easy to grow with controlled a length, diameter and density [3-5]. The application of structured (vertically aligned) MWCNTs were first investigated in [6], where the authors conducted an experiment to compare the contact resistances between an $\mathrm{Au}-\mathrm{Au}$ contact pair ( $\mathrm{Au}$ hemispherical ball and Au substrate) and an Au-MWCNT contact pair (Au hemispherical ball and MWCNT substrate). They found that the contact resistance of the Au-MWCNT contact pair was higher $(\sim 108 \Omega)$ than that of the $\mathrm{Au}-\mathrm{Au}$ contact pair $(\sim 0.58 \Omega)$ [6-9]. This greater contact resistance results from the nonconductive substrate on which MWCNT are grown. The substrate inhibits the current travelling along the MWCNTs; only lateral conduction is possible between the MWCNTs, resulting in higher contact resistance. To improve the performance a gold-coated surface (Au/MWCNT) was developed [6-15]. The early results show that a $\mathrm{Au} / \mathrm{MWCNT}$ surface has great potential for application as a switch contact surface. In [6], experiments were conducted to study the change in contact resistance between gold-coated MWCNTs and a gold-coated stainless steel hemispherical ball, (Au-Au/MWCNT). The contact resistance of the $\mathrm{Au}-\mathrm{Au} / \mathrm{MWCNT}$ contact pair was $0.68 \Omega$

Yunus et al. [13] observed that the use of Au/MWCNT as a contact surface prolongs the performance lifetime of the switch contacts. A Au-Au/MWCNT contact pair, with CNTs of $50 \mu \mathrm{m}$ height, was tested at current levels of 20 , 30,40 and $50 \mathrm{~mA}$, with a potential difference of $4 \mathrm{~V}$ and contact force of $1 \mathrm{mN}$. The results indicated cycles to failure of 70 million, 120,100 and 80 cycles $[9,10,12]$ for current levels of 20,30, 40 and $50 \mathrm{~mA}$; the results are replicated here as the solid line in Fig.8. The reference $\mathrm{Au}-\mathrm{Au}$ contact pair, maintained a stable contact resistance for 220 cycles for a current load of $10 \mathrm{~mA}, 4 \mathrm{~V}$ at $1 \mathrm{mN}$ static load. It was observed that the results at current 
levels $30-50 \mathrm{~mA}$ were dominated by a delamination process but at a current level at $20 \mathrm{~mA}$, the fine transfer process dominated [13-15].

The thermal model of fine transfer mechanism [12, 14] is used to evaluate the number of cycles to failure for a contact surface. A 3D confocal laser map of the worn $\mathrm{Au} / \mathrm{MWCNT}$ surface was used to determine the area of the removed material, $\left(\mathrm{A}_{\mathrm{r}}\right)$ from the $\mathrm{Au}$ film at the point of failure, defined by the rapid increase in the contact resistance to a magnitude three times the nominal value. The thickness of the Au film was assumed to be uniform, $(t)$. Thus the volume of material transferred per operation $(\Delta v)[12,14]$ :

$$
\Delta \mathrm{v}=\left(\mathrm{A}_{\mathrm{r}} \mathrm{t}\right) / \mathrm{N}
$$

Where, $\mathrm{N}$ is the number of switching operations at the point of failure. Thus the general relationship [12,14] can be expressed as,

$$
\Delta \mathrm{v}=\left(3.85 \times 10^{-10}\right) \mathrm{I}^{2}
$$

Where, I is current (A). It has since been observed that each switch closing operation consists of several bounces [14]. In the previous studies the bounce phenomenon had not been accounted for in calculating the cycles to failure. In addition, it is important to note that for the condition of $4 \mathrm{~V}$ there is no evidence of arcing, unlike the case for $12 \mathrm{~V}$ which is commonly used in metallic electrical contact surfaces $[16,17]$.

This paper presents further results to confirm the predicted data from the fine transfer model in equation (2). The sample contact pair is an Au hemispherical ball and Au/MWCNT, $30 \mu \mathrm{m}$ in height. In this paper the $\mathrm{Au}$ hemispherical ball is improved by adding a chromium layer to help the Au layer adhere on the $2 \mathrm{~mm}$ stainless steel ball. Moreover the relationship between the number of bounces and the contact resistances and the number of cycles to failure will be considered.

\section{Material preparation}

The contact consists of two surfaces which are classified according to the direction of the DC electric current. One surface is the cathode which for our setup is a $2 \mathrm{~mm}$ in diameter stainless steel hemispherical ball sputter coated with a $10 \mathrm{~nm}$ thick Chromium (Cr) layer on which $500 \mathrm{~nm}$ of $\mathrm{Au}$ is sputtered. The Au coated ball has a surface roughness of $\mathrm{Ra} \approx 92 \mathrm{~nm}$. The Cr layer acts as the adhesion layer to help the Au adhere on the stainless steel. The other surface forms the anode, which is the silicon wafer coated with MWCNTs and sputter coated with $\approx 500 \mathrm{~nm} \mathrm{Au}$. This Au/MWCNT surface has a surface roughness of $\mathrm{R}_{\mathrm{a}} \approx$
1,375 $\mathrm{nm}$. The MWCNTs growth is divided into three processes: cleaning, sputtering and growing as shown in Figure 1.

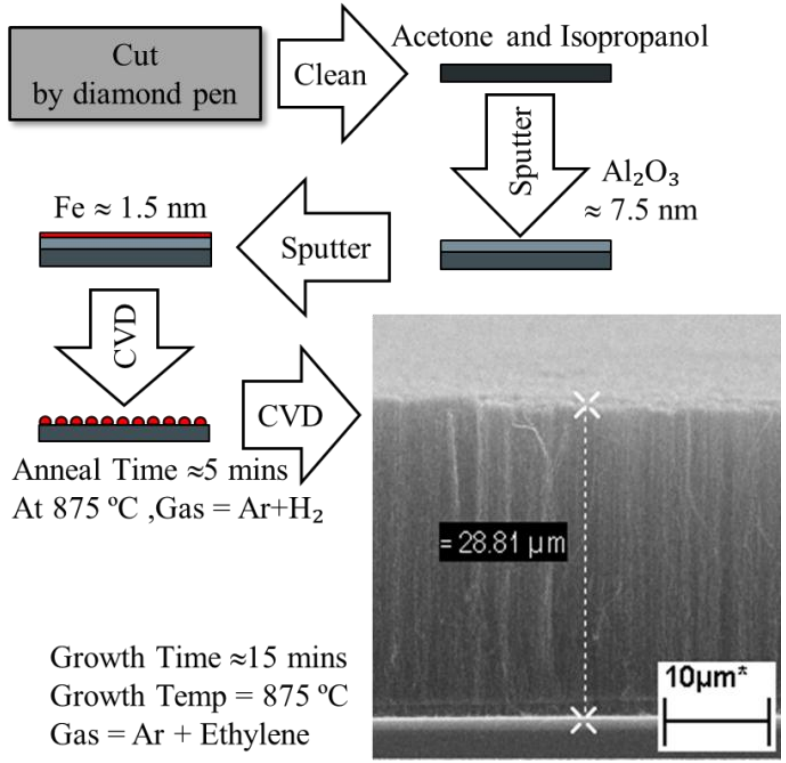

Figure 1. CNTs growth apparatus and procedure.

The cleaning process consists of submerging and sonificating the silicon wafers $(7 \mathrm{~mm}$ by $2 \mathrm{~mm}$ ) for 20 minutes in acetone and isopropanol. The clean substrate is then coated with a $7.5 \mathrm{~nm}$ thick $\mathrm{Al}_{2} \mathrm{O}_{3}$ buffer layer, on top of which a $1.5 \mathrm{~nm}$ thick Fe catalyst layer is sputtered. The last process is growing the MWCNTs which is accomplished using chemical vapor deposition (CVD). After the substrate is put in the center of the CVD furnace, both $\mathrm{Ar}$ and $\mathrm{H}_{2}$ gases are flowed into the chamber which is heated to $875{ }^{\circ} \mathrm{C}$ At this step, the substrate is annealed at this temperature for 5 minutes. The anneal step is needed to create $\mathrm{Fe}$ islands. These islands act as the seeds for MWCNT to grow. After the substrate is annealed, ethylene $\left(\mathrm{C}_{2} \mathrm{H}_{4}\right), \mathrm{H}_{2}$ and Ar flow through the chamber at $875^{\circ} \mathrm{C}$ for 15 minutes to form a MWCNT height of approximately 30 $\mu \mathrm{m}$. The final step in the fabrication of the Au/MWCNTs substrate is sputtering the MWCNT substrate with $500 \mathrm{~nm}$ of Au. The resulting substrate has a surface roughness of Ra $1,375 \mathrm{~nm}$ and Au penetration depth of around $1 \mu \mathrm{m}$ as shown in Figure 2.

\section{Methodology}

To simulate the repeated switching action of a MEMS relay, the Au/MWCNTs substrate is attached to a PZT cantilever. The test platform consists of the PZT with $\mathrm{Au} / \mathrm{MWCNT}$ substrate, the Au-coated hemispherical surface, mounted on an anti-vibration workstation, in a temperature-controlled room. In Figure 3, a schematic of 
the test system is shown. The dynamic force as shown in Figure 4 from a piezoelectric force sensor.

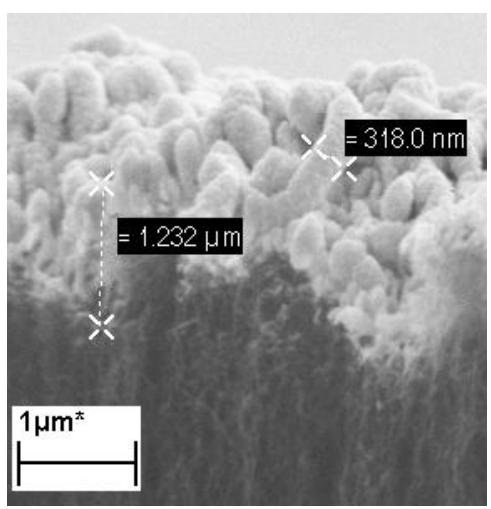

Figure 2. Au/MWCNT composite contact.

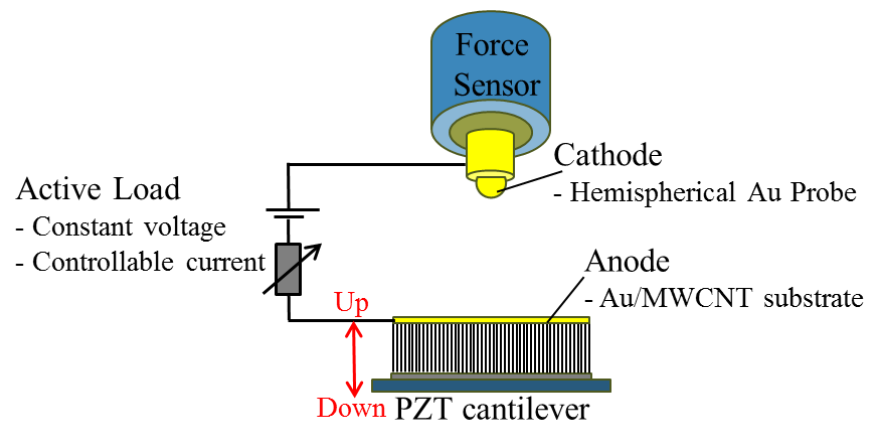

Figure 3. The schematic of electric contact configuration.

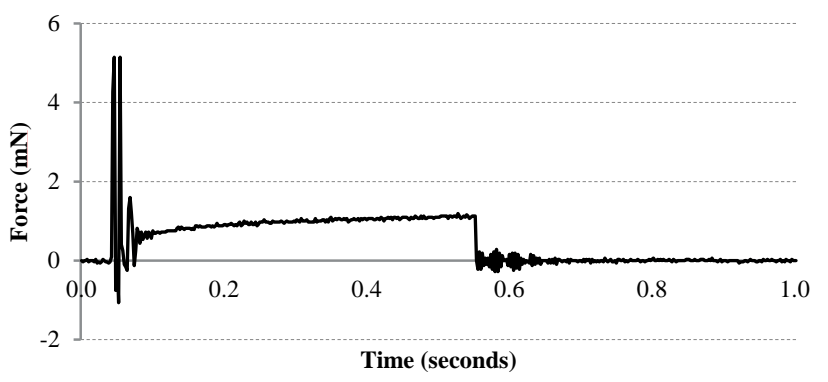

Figure 4. Measured Force.

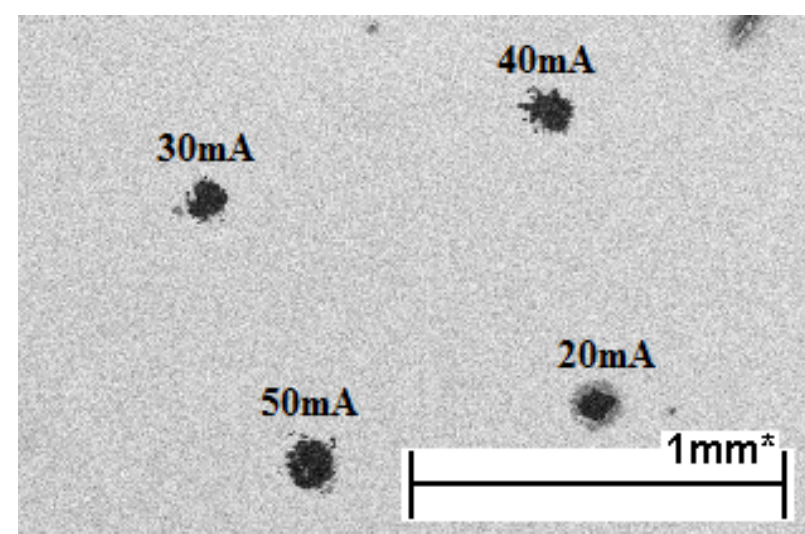

Figure 5. The Au/MWCNT substrate $(30 \mu \mathrm{m}$ in height)
The experiments were conducted with current levels of 20, 30, 40 and $50 \mathrm{~mA}$, a potential difference of $4 \mathrm{~V}$, and a nominal contact force $1 \mathrm{mN}$. All current levels were tested on the same sample but different positions to maintain consistency in the Au/MWCNT sample properties as shown in Figure 5. A signal function generator with voltage amplification actuates the PZT cantilever with a voltage level of $20 \mathrm{~V}$ at a frequency range of $1-100 \mathrm{~Hz}$. Testing is performed as follows:

1. Apply voltage to vibrate PZT cantilever by the function generator at testing frequency $\left(f_{\text {test }}\right)$.

2 . Record the number of bounces $\left(B_{c}\right)$ while testing.

3. Measure and record the contact resistance $\left(R_{c}\right)$ and the number of bounces at the $10^{\text {th }}, 100^{\text {th }}, 1000^{\text {th }}$, $10000^{\text {th }}$, and $100,000^{\text {th }}$ cycles, and then every 12 hours until the contact fails. Record time to failure $\left(\mathrm{t}_{\text {test }}\right)$.

For the experiment operated at $20 \mathrm{~mA}, \mathrm{f}_{\text {test }}$ was $30 \mathrm{~Hz}$. For $30-50 \mathrm{~mA}$, the $\mathrm{f}_{\text {test }}$ was set up at $100 \mathrm{~Hz}$ to reduce testing time. Therefore, the numbers of operations $\left(\mathrm{N}_{\text {test }}\right.$, cycles) are derived from;

$$
\mathrm{N}_{\text {test }}=\mathrm{t}_{\text {test }} \times \mathrm{f}_{\text {test }}
$$

As shown in Figure 4, the impacting force $(1 \mathrm{mN})$ from the PZT cantilever will be stable after the contact is closed for 0.2 seconds. Therefore, to measure the $B_{c}, f_{\text {test }}$ has to be decreased to $1 \mathrm{~Hz}$ before counting to get the complete bounce process. The contact resistance $R_{c}$ is measured using 4-wire measurement method. While measuring the $\mathrm{R}_{\mathrm{c}}$, the PZT cantilever is stopped for 5 minutes to obtain a stable reading.

\section{Results}

A. Contact resistance against the number of cycles

The contact resistance against the number of cycles is shown in Figure 6.

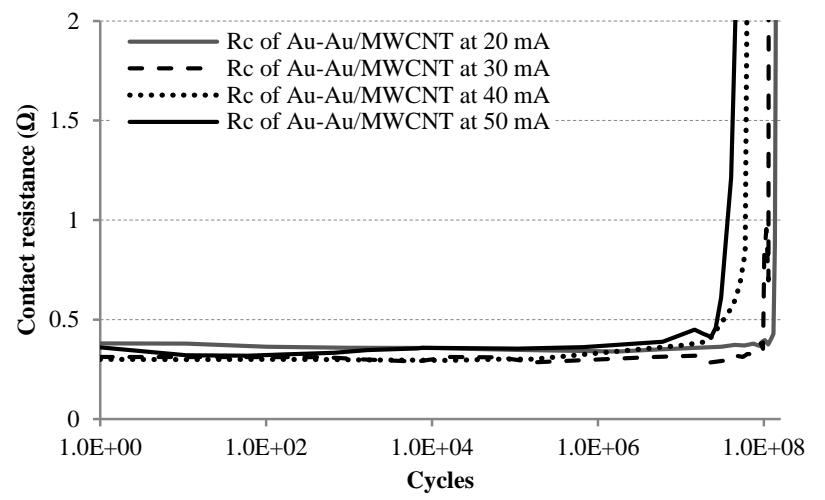

Figure 6. Contact resistance against number of cycles for Au-Au/MWCNT contact pair with current levels of 20-50 mA, 4 $\mathrm{V}$, nominal contact force $1 \mathrm{mN}$. 
The $R_{c}$ versus cycles to failure trend is similar across all values of current. The contact resistance behavior can be divided into four stages. The first stage is an unstable stage of $R_{c}$. The $R_{c}$ starts at below $0.4 \Omega$ and slowly decreased across 5,000 cycles. Next is the stable stage of $R_{c}$, where there is gradual increase in Rc with time. Then the switching contact goes through the rising stage of $R_{c}$. In this stage, the $R_{c}$ gradually goes up to the breakdown point. Then the contact is in the failure stage of $R_{c}$ which shows the abrupt increase to more than three times of the nominal resistance called contact failure.

B. The number of bounces against the number of cycles The number of bounce against the number of cycles is shown in Figure 7.

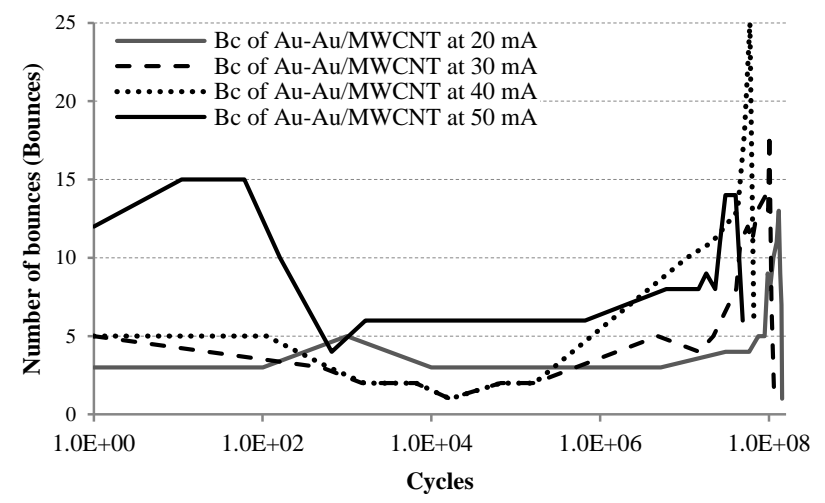

Figure 7. The number of bounce against number of cycles for Au-Au/MWCNT contact pair with current level of 20,30, 40, 50 $\mathrm{mA}, 4 \mathrm{~V}$, contact force $1 \mathrm{mN}$.

The graph compares the number of bounce $\left(B_{c}\right)$ between the experiments operated at the different current levels. The change in bounce behavior can be divided into four stages, as with the $R_{c}$ behavior. The first stage of $B_{c}$ is an unstable stage. The $\mathrm{Bc}$ starts at between 3-5 bounces except the experiment operated at $50 \mathrm{~mA}$ for which $B_{c}$ is 12. The reason for the distinct number of bounces is that the Au/MWCNT surface is usually not consistent for the whole substrate. Thus, the height, density and diameter of some points of Au/MWCNT surface may be different to other points. This results in the different number of bounces. After performing switching for 5,000 cycles, the $B_{c}$ was decreased to 1-4 bounces. This is the stable stage of $B_{c}$, which shows gradually increase in the number of bounces for this period. Then the switching contact shifts to the rising stage of $B_{c}$. In this stage, the $B_{c}$ rises sharply to the highest value. Finally, the contact is in the failure stage of $\mathrm{B}_{\mathrm{c}}$, which shows the rapid reduction, indicating that the contact has completely failed.

\section{Discussion}

The data from Figure 6 is used to obtain the numbers of cycles $\left(\mathrm{N}_{\text {test }}\right)$ to failure of the experiment at current levels 20, 30, 40 and $50 \mathrm{~mA}$ as shown in Table 1. These are defined as the number of cycles when $\mathrm{R}=1.5 \Omega$.

Table 1. The number of cycles $\left(\mathrm{N}_{\text {test }}\right)$ to failure of the experiment at current levels: 20, 30, 40 and $50 \mathrm{~mA}$.

\begin{tabular}{|c|c|c|c|}
\hline $\begin{array}{c}\text { Current } \\
(\mathbf{m A})\end{array}$ & $\begin{array}{c}\text { Fine transfer } \\
{[\mathbf{1 2}]}\end{array}$ & $\begin{array}{c}\text { Previous data } \\
{[\mathbf{1 2}]}\end{array}$ & $\begin{array}{c}\text { Experiment } \\
\text { data, } \mathbf{N}_{\text {test }}\end{array}$ \\
\hline 1 & $2.8 \mathrm{E}+10$ & - & - \\
\hline 10 & $2.8 \mathrm{E}+08$ & - & - \\
\hline 20 & $7.0 \mathrm{E}+07$ & $7.0 \mathrm{E}+07$ & $1.4 \mathrm{E}+08$ \\
\hline 30 & $3.1 \mathrm{E}+07$ & $2.0 \mathrm{E}+02$ & $1.2 \mathrm{E}+08$ \\
\hline 40 & $1.8 \mathrm{E}+07$ & $1.0 \mathrm{E}+02$ & $6.6 \mathrm{E}+07$ \\
\hline 50 & $1.1 \mathrm{E}+07$ & $8.0 \mathrm{E}+01$ & $4.9 \mathrm{E}+07$ \\
\hline
\end{tabular}

$\mathrm{N}_{\text {test }}$ can be plotted for comparison with the predicted data from the fine transfer model [12] and the results from previous research [12] as shown in Figure 8. This shows that the addition of the $\mathrm{Cr}$ layer under the Au surface on the ball has stabilized the surfaces for the higher current levels above $>20 \mathrm{~mA}$. It is also apparent that for the only data point that is common, (20 mA) the new sample has undergone twice as many cycles before failure.

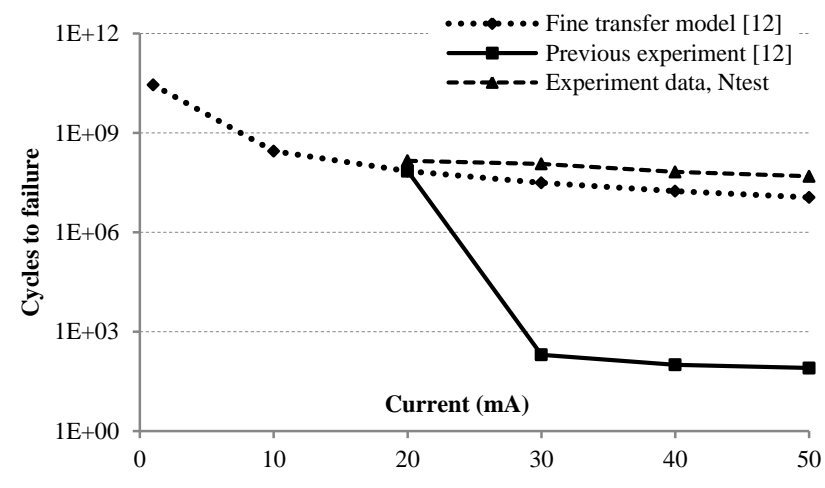

Figure 8. The number of cycle to failure for a given load current (4 V, mA). Solid line is experimental data. Dashed line is predicted data from fine transfer model [12]. Dotted line is the previous research results [12].

Since the trends of the $\mathrm{R}_{\mathrm{c}}$ and $\mathrm{B}_{\mathrm{c}}$ with current are similar, the data for $30 \mathrm{~mA}, 4 \mathrm{~V}(1 \mathrm{mN})$ is chosen to show the graph appearance of the four stages of the failure. These four stages namely unstable, stable, rising and failure stages as shown in Figure 9. The first stage is the unstable stage. In Figure 10, the decreasing trend of both contact resistance and number of bounce is shown. The MWCNT will buckle and deformed permanently if the contact force is greater than a load threshold, resulting in plastic deformation. Based on the observation, this permanent 
deformation process of the Au/MWCNT substrate with 30 $\mu \mathrm{m}$ MWCNT is completely finished within 5,000 switching cycles.

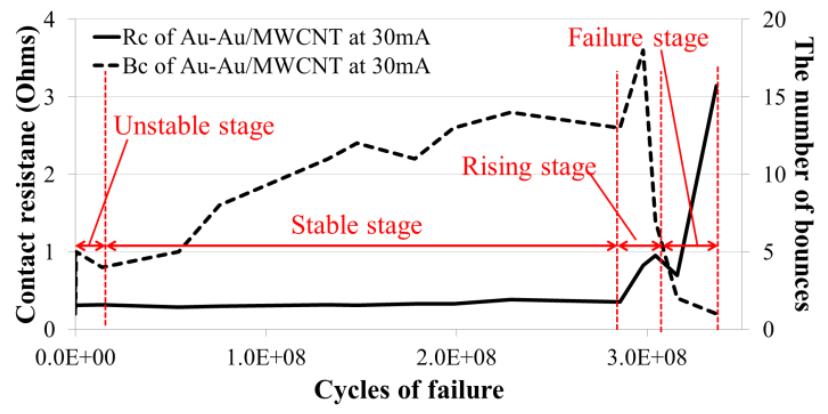

Figure 9. Four stages of the contact resistance and the number of bounces behavior.

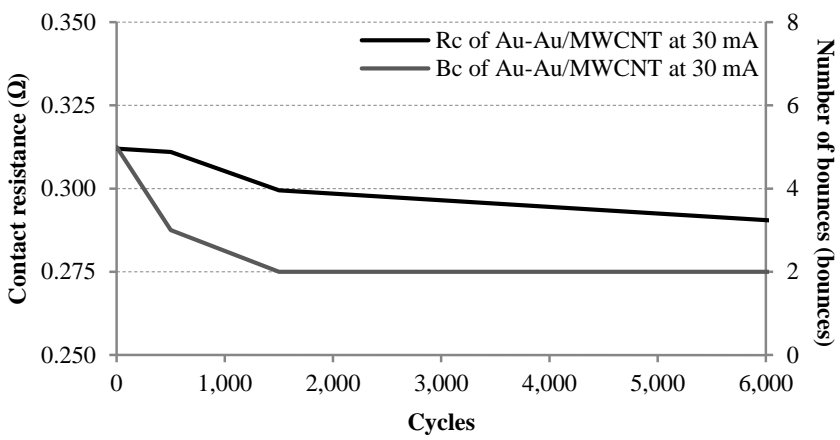

Figure 10. The unstable stages of the failure behavior.

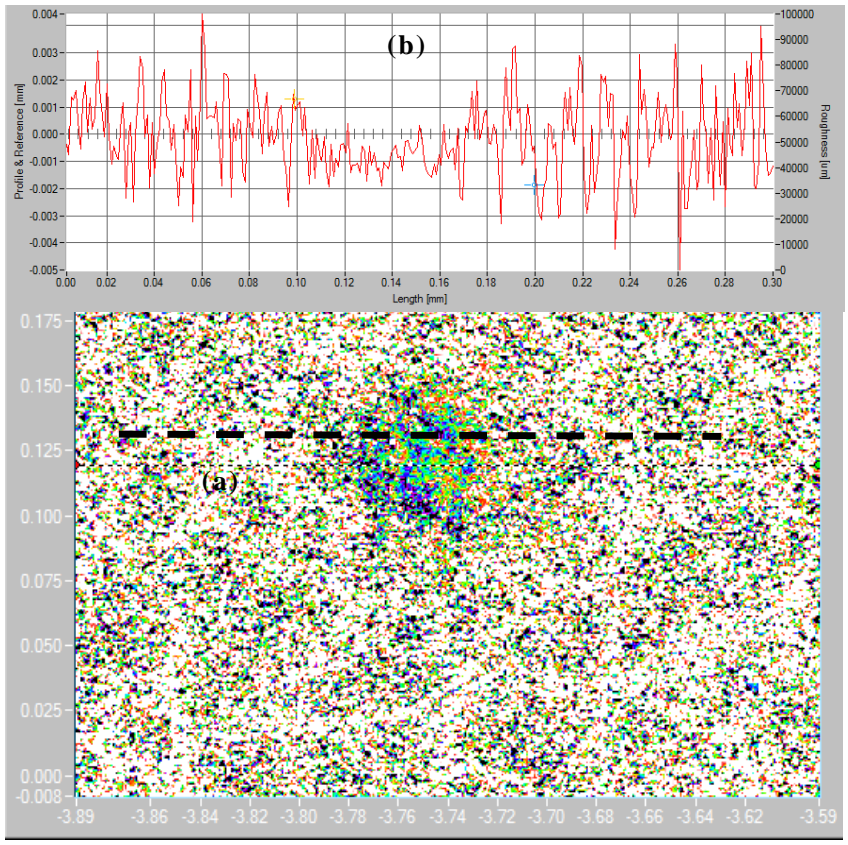

Figure 11. (a) 3D surface profile of anode surface, 301X301 data points over area $0.3 \times 0.3 \mathrm{~mm}$. of Au-Au/MWCNT; $30 \mu \mathrm{m}$ height,

(b) Surface profile of Au-Au/MWCNT composite surface.

To investigate the wear-in period further, the
$\mathrm{Au}-\mathrm{Au} / \mathrm{MWCNT}$ contact pair was tested for around 5,000 cycles with a current level of $30 \mathrm{~mA}, 4 \mathrm{~V}(1 \mathrm{mN})$. After which a laser profiler was used to scan the Au/MWCNT surface (Figure 11 (a)) to investigate the profile of the crater of the MWCNT deformation, as shown in Figure 11 (b).

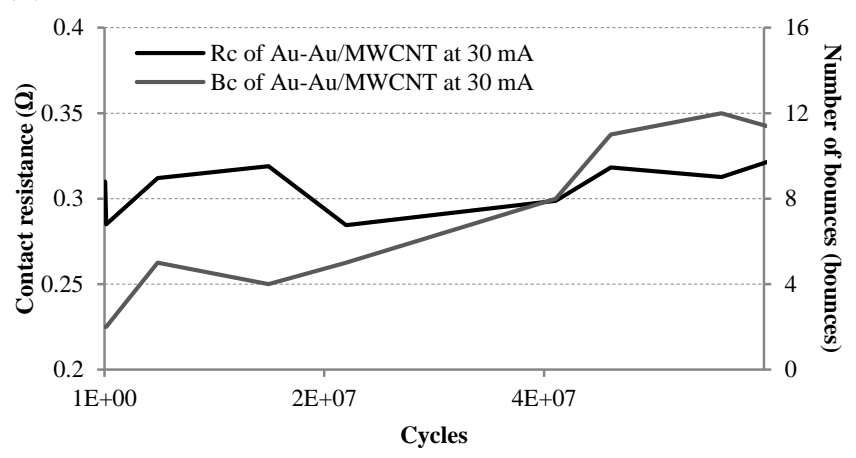

Figure 11. The stable stage.

The stable stage is plotted over 40 Million cycles and shows a small increase in the number of bounces, and a stable contact resistance. The $R_{c}$ and $B_{c}$ are stable within the small ranges $\left( \pm 0.05 \Omega\right.$ for $R_{c}$ and 4-8 bounces for $\left.B_{c}\right)$ as shown in Figure 11. At this stage, the fine transfer mechanism is the predominant transfer process. The $\mathrm{Au}$ layer is gradually removed from Au/MWCNT (cathode) to Au probe (anode). This leads to a gradually change in the contact surface. To confirm this, an experiment with $\mathrm{Au}-\mathrm{Au} / \mathrm{MWCNT}$ surface of $30 \mu \mathrm{m}$ height, with a current level of $30 \mathrm{~mA}, 4 \mathrm{~V}(1 \mathrm{mN})$ was tested for around 1 million cycles. The SEM was then used to investigate the contact surface as shown in Figure 12. It can be observed that $\mathrm{Au}$ layer on the $\mathrm{Au} / \mathrm{MWCNT}$ surface is starting to deplete and transfer to the Au ball.

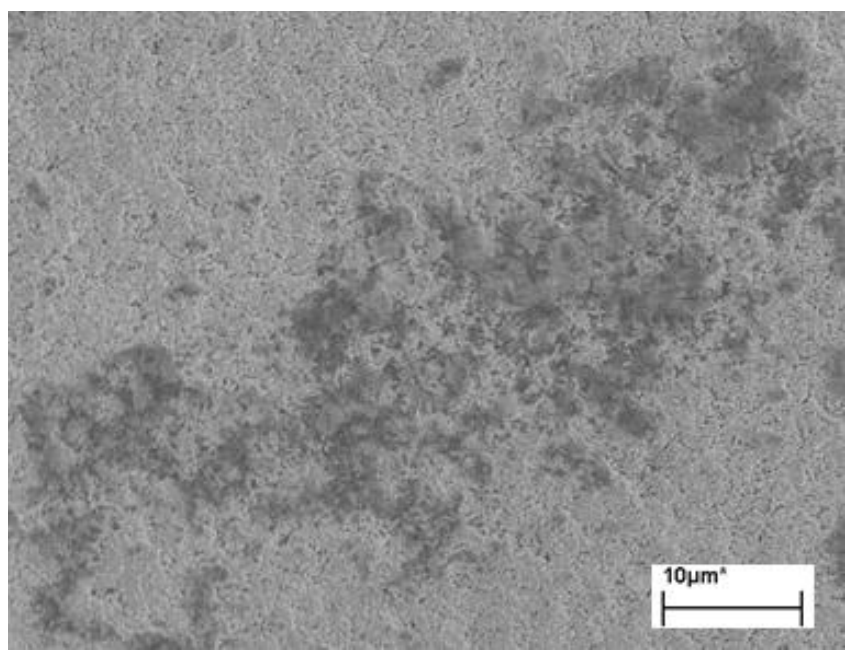

Figure 12. Au layer transfer at 1 million cycles (yet to fail) on the Au/MWCNT surface, $30 \mu \mathrm{m}$ height.

When the switching contact is in the rising stage, the 
contact resistance and number of bounces continues to rise, albeit with a steeper gradient. The depleted surface causes the increase in $\mathrm{Au}$ the contact resistance due to the decrease in the metallic contact area. Previously the current passes through the $\mathrm{Au}$ asperities at the centre of the surface. When the centre area does not have any Au surface remaining because of the material transfer process, the current density will travel through the area surrounding centre until the contact is completely worn out (i.e. contact failure). To illustrate this process, the same experiment was tested to 27 million cycles. Then the SEM was used to examine the contact surface as shown in Figure 13. The Au layer is completely depleted at the centre as shown in the black area. The current starts to move via the area around the probe as shown in grey around the depleted area. Therefore, the conductive area supporting the current density is only the grey shaded area.

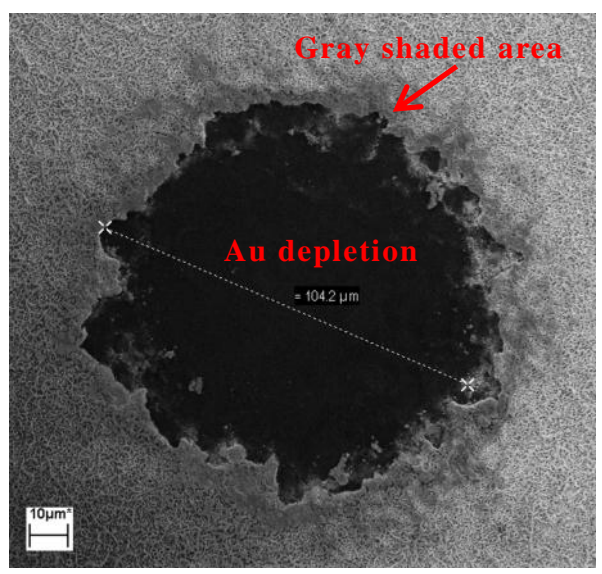

Figure 13. Material trasfer at 27 th million cycle (not fail) on the $\mathrm{Au} / \mathrm{MWCNT}$ surface; $30 \mu \mathrm{m}$ height.

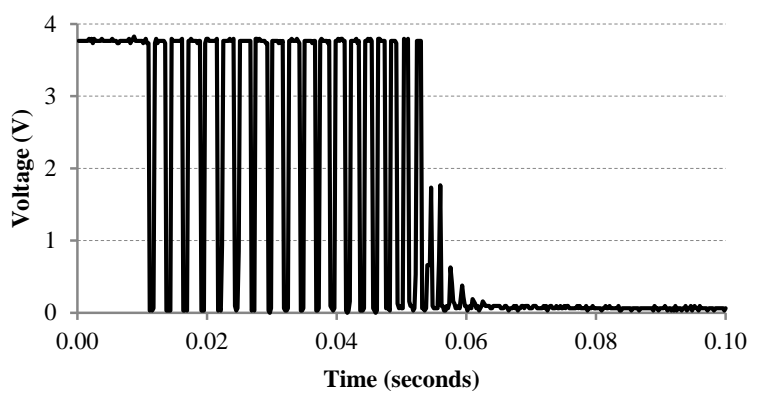

Figure 14. The bounce process at the highest point on the rising stage

In Figure 13, the MWCNT is exposed in the black area. It is known that CNTs have high elastic moduli [2,3] and so the number of bounces should increase with gold removal. The number of bounces increases sharply to the maximum number of bounces when the Au layer nearly completely worn out. The maximum bounce process is shown in Figure 14.

The final stage is the failure stage of $R_{c}$, which shows the contact resistance rising rapidly. In this stage the contact surface is totally depleted as shown in Figure 15, i.e. the contact area completely touches the MWCNT underneath. The MWCNT surface causes the high contact resistance of this last stage. Therefore, the contact pairs are considered to have failed as the contact resistance is greater a magnitude of three times the nominal value [12], which in this case is about $1.5 \Omega$.

The number of bounce drops rapidly in the failure stage to show that the contact has failed. At this stage, the number of bounces should be equal to the peak amount in the rising stage. However, since the contact surface is almost totally depleted, some bounce events are not recorded as shown in Figure 17 as the switch touches the non-conductive area which is entirely MWCNT.

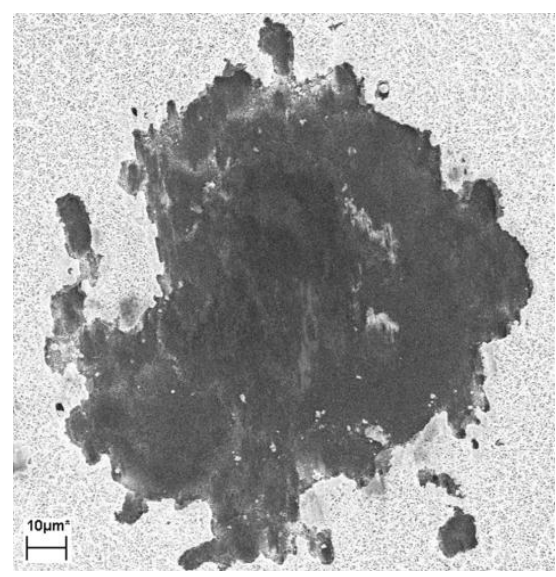

Figure 15. Au/MWCNT surface at 49 million cycles (fail contact) tested at current level of $30 \mathrm{~mA}$.

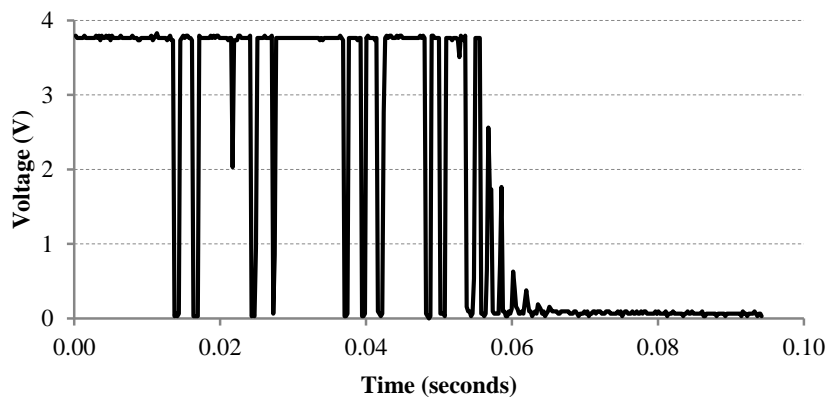

Figure 16. The bounce process in the failure stage for $\mathrm{Au}-\mathrm{Au} / \mathrm{MWCNT}, 30 \mu \mathrm{m}$ height contact pair with current level of $30 \mathrm{~mA}, 4 \mathrm{~V}(1 \mathrm{mN})$.

In summary, it is possible to use the number of bounces to predict the failure of the switch contact. Thus far, theoretical model has been developed to predict the exact number of bounces because $B_{c}$ depends on many 
influences such as the height of MWCNT and the Au thickness. However, a benefit of the results presented that it allows the trend of $B_{c}$ to be used monitoring if the contact is going to fail, i.e. when the number of bounces is increases sharply.

\section{Conclusion}

It has been shown that an Au ball surface coupled with an Au/MWCNT (gold-coated multi-walled carbon nanotube structure) substrate, can withstand more than 49 million switching cycles at $50 \mathrm{~mA}(0.2 \mathrm{~W})$, at a low contact force of $1 \mathrm{mN}$. This is a significant increase in current over previous published work on these surfaces. The contact resistance across the interface is used as the indicator for determining the failure modes of the contact switch.

The following conclusions are reached:

(1) The contact resistance trend is similar to the previous studies, and remains stable for over 49 million switching cycles for all current levels between 20 and $50 \mathrm{~mA}$.

(2) The change of height of Au/MWCNT from 50 to 30 $\mu \mathrm{m}$ does not change the applicability of the fine transfer model.

(3) The mechanism of failure previously defined as fine transfer shows that the Au-MWCNT anode surface is losing material to the cathode ball.

(4) The results show that the orientation of the contact surfaces is not significant. In this case the Au ball anode is installed above Au/MWCNTs cathode substrate, unlike the previous results in [10]. Referring to the experimental set up, gravity does not affect the material transfer.

(5) The contact bounce is affected by the changes in the surface and is correlated with the changes in $R_{c}$.

(6) The trend of number of bounces trend can be used to predict the lifetime. If the contact resistance or the number of bounces starts to increase rapidly, the user can predict that the contact is going to fail in the near future.

\section{References}

1. R.A. Jr.Coutu, et al., Microswitches with sputtered $A u$, $A u P d, A u$-on-AuPt, and AuPtCu alloy electric contacts. Components and Packaging Technologies (2006), 29: pp. 341-9.

2. M. S. Dresselhaus, G. Dresselhaus, and P. Avouris Carbon Nanotubes: Synthesis, Structure, Properties, and Applications. ed. by 1, Berlin: Springer (2001).
3. A. Jorio, G. Dresselhaus, and M.S. Dresselhaus, Carbon Nanotubes: Advanced Topics in the Synthesis, Structure, Properties and Applications. ed. by 1. Vol. 111, Applied Physics Springer (2008).

4. V. Sgobba and D.M. Guldi, Carbon nanotubes-electronic/electrochemical properties and application for nanoelectronics and photonics. Chemical Society Reviews (2009), 38: pp. 165-184.

5. W. Maser, A.M. Benito, E. Munoz, and M.T. Martinez, Carbon Nanotubes: From Fundamental Nanoscale Objects Towards Functional Nanocomposites and Applications. ed. by 1 (Netherlands: Springer, 2008).

6. E.M. Yunus, J.W. McBride, and S.M. Spearing, 'The Relationship between Contact Resistance and Contact Force on Au Coated Carbon Nanotube Surfaces', in The 53rd IEEE Holm Conference on Electrical Contacts (USA: Institute of Electrical and Electronics Engineers, 2007), pp. 167-74.

7. E.M. Yunus, J.W. McBride, and S.M. Spearing, 'Improving the Contact Resistance at Low Force Using Gold Coated Carbon Nanotube Surfaces', in The 24th International conference on electrical contact (St Malo, France, 2008), pp. 507-13.

8. E.M. Yunus, J.W. McBride, and S.M. Spearing, 'Investigation of Gold Sputter Coated Vertically Aligned Multi-Walled Carbon Nanotubes for Rf Mems Contact Surfaces', in The Material Research Society Meeting, Fall Meeting Symposium GG. (Warrendale, USA, 2008).

9. E.M. Yunus, J.W. McBride, and S.M. Spearing, The Relationship between Contact resistance and Contact Force on Au Coated Carbon Nanotube Surfaces Under Low Force Conditions. Components and Packaging Technologies (2009), pp. 650-657.

10.E.M. Yunus, J.W. McBride, and S.M. Spearing, Improving the Contact Resistance at Low Force using Gold Coated Carbon Nanotube Surfaces. European Physical Journal - Applied Physics, 2010. 50(1): p. 12904-12909.

11. E.M. Yunus, J.W. McBride, and S.M. Spearing, 'Low Force Electrical Switching Using Gold Coated Vertically Aligned Multi-Walled Carbon Nanotubes Surfaces', in IEICE Technical Report (Sendai, Japan,: International Session on Electro-Mechanical Devices, 2008), pp. 61-64.

12. J.W. McBride, The wear processes of gold coated multi-walled carbon nanotube surfaces used as electrical contacts for micro-electro-mechanical 
switching. Nanoscience and Nanotechnology Letters (2010), 2(4): pp. 357-361.

13. J.W. McBride, E.M. Yunus, and S.M. Spearing, 'Gold Coated Multi-Walled Carbon Nanotube Surfaces as Low Force Electrical Contacts for Mems Devices: Part 1', in Proceedings of the 55th IEEE Holm Conference on Electrical Contacts (Minneapolis, USA, 2009), pp. 281-87.

14. J.W. McBride, S.M. Spearing, L. Jiang, and C. Chianrabutra, 'Gold Coated Carbon-Nanotube Surfaces as Low Force Electrical Contacts for MEMS Devices: Part II, Fine Transfer Mechanisms', in The 57th IEEE Holm Conference on Electrical Contacts (Minneapolis, USA: Institute of Electrical and Electronics Engineers, 2011), pp. 1-6.

15. J.W. McBride, L. Jiang, and C. Chianrabutra, 'Fine Transfer in Electrical Switching Contacts Using Gold Coated Carbon-Nanotubes', in The 26th International Conference on Electrical Contact (Beijing, China, 2012), pp. 353-58.

16. J.F. Llewellyn, The Physics of Electrical Contacts. ed. by 1, Oxford Clarendon Press (1957).

17. R. Holm, Electric Contacts: Theory and Application. ed. by 4 , Springer (1999). 